\title{
The Unrested Resting Brain: Sleep Deprivation Alters Activity within the Default-mode Network
}

\author{
Ninad Gujar ${ }^{1}$, Seung-Schik Yoo $^{2}$, Peter $\mathrm{Hu}^{1}$, and Matthew P. Walker ${ }^{1}$
}

\begin{abstract}
The sleep-deprived brain has principally been characterized by examining dysfunction during cognitive task performance. However, far less attention has been afforded the possibility that sleep deprivation may be as, if not more, accurately characterized on the basis of abnormal resting-state brain activity. Here we report that one night of sleep deprivation significantly disrupts the canonical signature of task-related deactivation, resulting in a double dissociation within anterior as well as posterior midline regions of the default network. Indeed, deactivation within these regions alone discriminated sleep-deprived from sleep-control
\end{abstract}

\section{INTRODUCTION}

To date, the sleep-deprived brain has been investigated by examining alterations in activation during performance on a number of cognitive tasks. These productive studies have revealed alterations (both impairments and potential compensations) during tests of working memory, attention, monitoring, decision making, memory encoding, and failed off-line consolidation (Chee \& Chuah, 2008; Walker \& Stickgold, 2006; Drummond \& Brown, 2001). Although such studies provide critical insights into the neural disparities occurring during task performance, little attention has been given to the possibility that sleep deprivation maybe equally well described on the basis of differences in resting-state modes of brain activation.

The concept of a structured and constant state of "defaultmode" brain activity continues to gain considerable empirical support (Buckner, Andrews-Hanna, \& Schacter, 2008). One method for evaluating this default mode has been the mapping of networks in which activity is greater during rest or "baseline" state than during an experimental task, also described as task-induced deactivation (Raichle \& Snyder, 2007; McKiernan, Kaufman, Kucera-Thompson, \& Binder, 2003a; Mazoyer et al., 2001; Binder et al., 1999; Shulman, Fiez, et al., 1997). A wealth of evidence has now identified a common set of brain areas-especially cortical midline structures of the posterior cingulate cortex (PCC), precuneus $(\mathrm{PrC})$, and medial $\mathrm{pFC}$, together with medial-

${ }^{1}$ University of California, Berkeley, ${ }^{2}$ Harvard Medical School subjects with a 93\% degree of sensitivity and $92 \%$ specificity. In addition, the relative balance of deactivation within these default nodes significantly correlated with the amount of prior sleep in the control group (and not extended time awake in the deprivation group). Therefore, the stability and the balance of task-related deactivation in key default-mode regions may be dependent on prior sleep, such that a lack thereof disrupts this signature pattern of brain activity, findings that may offer explanatory insights into conditions associated with sleep loss at both a clinical as well as societal level.

temporal lobe and bilateral inferior parietal cortex-that undergo task-induced deactivation (Buckner et al., 2008; Fransson \& Marrelec, 2008; Fox \& Raichle, 2007; Gusnard, Akbudak, Shulman, \& Raichle, 2001; Raichle et al., 2001; Shulman, Corbetta, et al., 1997).

Although the functional significance of coherent restingstate oscillations, including the default network, continues to be investigated (Balduzzi, Riedner, \& Tononi, 2008; Buckner et al., 2008; Buckner \& Vincent, 2007; Raichle \& Snyder, 2007), regulation of default-mode activity appears to be important for deploying the appropriate ontask networks necessary for optimal cognitive performance (Dosenbach et al., 2007; McKiernan, Kaufman, KuceraThompson, \& Binder, 2003b). Interestingly, perturbations in default-mode activity during wakefulness-including midline brain regions-have been identified in a number of disorders that display co-occurring abnormalities of sleep, including Schizophrenia (Garrity et al., 2007), autism spectrum disorders (Cherkassky, Kana, Keller, \& Just, 2006), anxiety disorders (Zhao et al., 2007), attention deficit disorder (Castellanos et al., 2007; Tian et al., 2006), and Alzheimer's disease (Buckner et al., 2008; Sorg et al., 2007; Greicius, Srivastava, Reiss, \& Menon, 2004), deficits that correlate with clinical and cognitive characteristics of the disease. Advancing this connection, it has been demonstrated that aspects of the default-mode network persist in their activity during sleep (Horovitz et al., 2008; Fukunaga et al., 2006), which may indicate that some operations of this default system continue during sleep, or that sleep modulates these networks, maintaining their next-day functionality. 
Here we test the hypothesis that the integrity of activity within this default-mode network is dependent on a night of prior sleep. We examine (a) whether one night of sleep deprivation disrupts the canonical signature of task-induced deactivation, (b) if differences in these deactivations are related to on-task trial success, and (c) whether the extent of such alterations are related to the amount of prior sleep or duration of extended time awake.

\section{METHODS}

\section{Participants}

A total of 28 healthy subjects, divided equally between males and females, aged 18-30 years (mean $=22.3$ years, $S D=2.8$ years), were randomly assigned to either the sleep-rested or the sleep deprivation conditions (although 2 of 14 sleep-rested participants were omitted from the analysis due to MRI data corruption). Subjects abstained from caffeine and alcohol for $72 \mathrm{hr}$ before and during the entire course of the study and kept a normal sleepwake rhythm and average sleep duration (7-9 hr of sleep per night, with morning wake time between 6:00 and 9:00 a.m.) for a period of 1 week before participation in the study, verified by sleep logs and actigraphy (an ambulatory wristwatch that senses movement and can distinguish between wake and sleep states). Subject exclusion criteria included a history of neurologic, psychiatric, or sleep disorders, a past history of drug abuse, and a current use of antidepressant or hypnotic medications.

\section{Experimental Procedures}

Subjects performed an incidental memory encoding task during an event-related fMRI scanning session at 6:00 p.m. $( \pm 1 \mathrm{hr}$ ) on Day 2 of the experimental protocol and completed a surprise recognition test following two nights of recovery sleep, allowing identification of successful (hits) and unsuccessful (misses) encoding trials. Changes in "on-task" brain activation and performance for the same subjects have been reported separately (Yoo, Hu, Gujar, Jolesz, \& Walker, 2007), describing independent and nonoverlapping results to those reported here. In the current report, we focus instead on differences in task-induced deactivation between groups.

The experimental manipulation differentiating the two conditions occurred on the night before the fMRI scanning session. Subjects in the sleep-rested group were awake across Day 1 and slept normally at home across Night 1, before returning for the scanning session on Day 2, and were asked to obtain $>7 \mathrm{hr}$ of sleep (although the final range of sleep times was substantive, as noted in the Results section). Subjects in the sleep deprivation condition were similarly awake across Day 1 but were subsequently kept awake across Night 1 and across Day 2, accumulating a mean of $35.2 \mathrm{hr}(S D=0.95 \mathrm{hr})$ of prolonged wakefulness before the scanning session. In contrast, subjects in the sleep-rested group obtained a mean of $7.8 \mathrm{hr}(S D=$ $1.42 \mathrm{hr}$ ) of sleep across the night before scanning session, as measured using sleep log diaries and cross validated with actigraphy recordings.

In the sleep deprivation group, subjects were continuously monitored throughout the enforced waking period by trained personnel in the laboratory and independently confirmed using actigraphy monitoring. During this time, subject activities were limited to Internet, e-mail, short walks, reading, and playing board games, providing a standardized regiment of waking activity.

\section{Scanning Session and Task}

The event-related fMRI session involved the presentation of 150 picture slides, subdivided into five randomized counterbalanced runs of 30 picture trials (for detailed methodology, see Yoo et al., 2007). Each trial contained a period of passive visual fixation, representing the control epoch of resting-state activity. During the "on-task" phase, subjects were asked to view the picture stimuli and make keypad responses indicating whether the picture was an indoor or outdoor scene. This enabled confirmation of stimulus viewing and also offered a cogent behavioral marker that subjects remained awake throughout the scanning session - a technique used in previous imaging studies of sleep deprivation (Drummond et al., 2000).

Each trial or "event" lasted 11 sec and began with a fixation crosshair (400-800 msec jittered), followed by the target picture for $2500 \mathrm{msec}$, during which subjects viewed the stimulus. Following this stimulus event, subjects were shown a screen with open squares for $2500 \mathrm{msec}$, indicating that subjects should make their response using a right-handed button-press. The trial was completed by a 4700- to 5100-msec of fixation baseline (equating jitter time). Analysis focused on this fixation-baseline epoch, which was contrasted with the on-task epoch, thereby allowing for the characterization of task-related deactivation in each group (Davis, Dennis, Daselaar, Fleck, \& Cabeza, 2008; Schilbach, Eickhoff, Rska-Jagiela, Fink, \& Vogeley, 2008; Fair et al., 2007; Dosenbach et al., 2006).

Stimuli were presented via MRI-compatible LCD goggles, and responses were obtained through a fiber-optic MRcompatible button box (Current Designs, Inc. Philadelphia, PA). Omitted trials were modeled as separate events and were not included in the calculation of resting-state activity. RTs for each trial were included in the design matrix as parametric regressors (Buchel, Holmes, Rees, \& Friston, 1998). This parametric modulation allowed the elucidation of brain activation that covaries with these regressors (here, RTs) at an individual level, resulting in conditionspecific activation that is independent of RTs.

\section{fMRI Procedures}

Functional imaging was performed on a General Electric (Waukesha, WI) 3-T magnet. Functional images were first 
acquired using an EPI sequence $(64 \times 64$ matrix, repetition time $=2500 \mathrm{msec}$, echo time $=40 \mathrm{msec}$, field of view $=$ $240 \mathrm{~cm}$, oblique slice parallel to AC-PC line, 34 slices, no slice gap, 4-mm thickness), followed by high-resolution T1-weighted structural images (three-dimensional spoiled gradient-echo sequence $=256 \times 192$ matrix, repetition time $=20 \mathrm{msec}$, echo time $=$ minimum, flip angle $=30^{\circ}$, field of view $=240 \mathrm{~cm}, 124$ slices, $1.3-\mathrm{mm}$ thickness). Scanner noise was reduced with MR-compatible headphones (Avotec, Stuart, FL), and head motion was minimized with foam pads.

\section{fMRI Analysis}

Preprocessing and data analysis were performed using Statistical Parametric Mapping software implemented in Matlab (SPM2; Wellcome Department of Cognitive Neurology, London, UK). Images were slice-timing corrected and motion-corrected and then spatially normalized to the Montreal Neurological Institute template and smoothed using an 8-mm FWHM Gaussian kernel. For each subject, task-induced deactivation was assessed by convolving a vector of the epoch onsets with a canonical hemodynamic response function HRF. A general linear model (GLM; Friston et al., 1995) was specified for each participant, resulting in the generation of respective parameter estimates. Statistical parametric maps were created for each subject by applying linear contrasts to the parameter estimates for these events of interest (Friston et al., 1998), resulting in a $t$ statistic for every voxel, contrasted to the on-task stimulus presentation epoch.

A whole-brain random effects analysis was performed to assess group differences in task-induced deactivation. Between-group comparisons were tested for using twosample $t$ Tests at a significance level $p<.001$, uncorrected, and $\geq 5$-voxel cluster size, a threshold used in prior taskinduced deactivation investigations (Davis et al., 2008; Damoiseaux et al., 2007; Sorg et al., 2007; Otten \& Rugg, 2001a) and consistent with our a priori focus on interest in this set of specific default ROIs. Activation maps were visualized using the Caret software (http://brainmap.wustl. edu/caret/) and the MRIcron software (http://www.sph.sc. edu/comd/rorden/mricron/).

To identify the capability of fMRI activity in categorizing those in the sleep-deprived group from those in sleeprested group, we use Receiver Operator Characteristic (ROC) methods (Fawcett, 2006). ROC calculations are useful for organizing classifiers and examining their ability to differentiate populations and are commonly used in clinical medicine for investigating the diagnostic power of specific variables in categorically separating disease groups (Linden, 2006). Using fMRI signals, we applied predictions from this technique to determine whether patterns of taskinduced deactivation were capable of accurately separating sleep-deprived subjects from sleep-rested subjects. In short, for a given a classifier (e.g., brain activation signal) and an instance (e.g., identification of a sleep-deprived subject), there are four possible outcomes. If the instance is positive (e.g., it is a sleep-deprived subject) and it is classified as positive, it is counted as a true positive; however, if it is classified as negative, it is counted as a false negative. If the instance is negative (e.g., not a sleep-deprived subject) and it is classified as negative, it is counted as a true negative; however, if it is classified as positive, it is counted as a false positive. From these outcomes, a measure of sensitivity (e.g., ability for identifying sleep-deprived subjects) and specificity (e.g., ability to exclude sleep-rested subjects) can be calculated (Fawcett, 2006).

\section{RESULTS}

Before comparing task-induced deactivation between the sleep-rested and the sleep-deprived groups, we first examined these patterns within each group separately; that is, mapping of brain networks in which activation was greater during the baseline fixation period than during the experimental task. As shown in Figure 1, both groups demonstrated significant task-induced deactivation in a distributed set of areas commonly reported as forming the archetypal default-mode network (Shulman, Corbetta, et al., 1997). These included midline ACC and PCC and bilateral superior parietal cortices together with bilateral medial-temporal lobe regions (coordinates provided in Table $1 \mathrm{~A}$ and $\mathrm{B}$, with lateral surface renderings provided in Supplementary Figure 1).

Next, we contrasted these patterns of task-induced deactivation between the two groups, thereby identifying the differential consequence of sleep deprivation, relative to the sleep-rested condition. This comparison revealed a marked alteration in default-mode activity between the two groups in the dorsal ACC (dACC) and the PrC regions

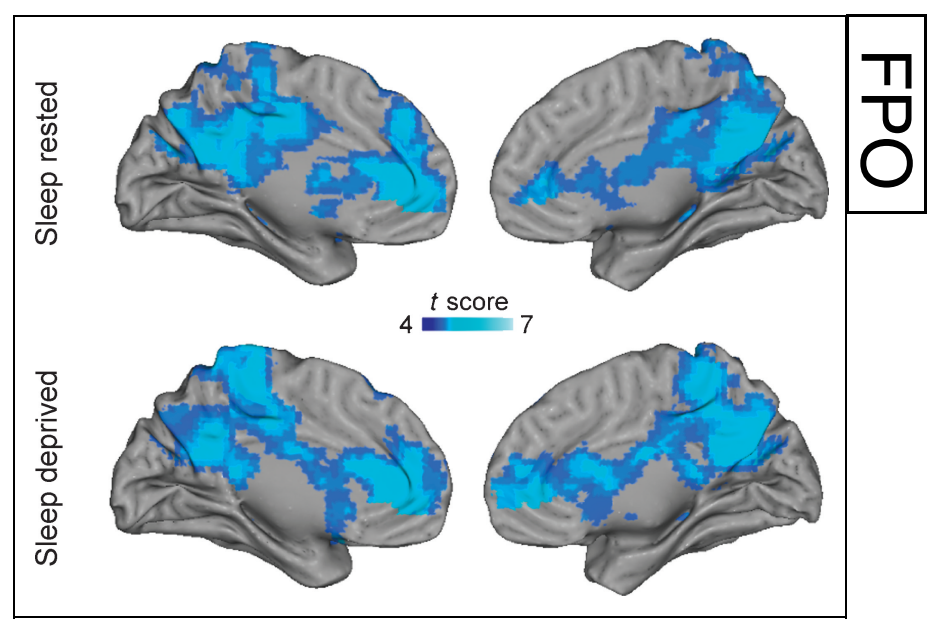

Figure 1. Regions of significant task-induced deactivation in the sleep-rested and sleep-deprived groups separately (full coordinates provided in Table 1A and B). Panels display deactivation on medial rendered surface brains for left and right hemispheres (lateral surfaces shown in Supplementary Figure 1). Effects are significant at $p<.001 ; \geq 5$ contiguous voxels 
Table 1. Anatomical Coordinates of Significant Clusters of Activation during Resting Fixation Period (Relative to Task Stimulus Viewing) within the Sleep Control Group and the Sleep Deprivation Group

\begin{tabular}{|c|c|c|c|c|c|}
\hline Region (BA) & Cluster Size (Voxels) & $x$ & $y$ & $z$ & Peak Z Score \\
\hline \multicolumn{6}{|l|}{ (A) Sleep Control Group } \\
\hline \multirow[t]{2}{*}{ Medial frontal gyrus (BA 10) $-\mathrm{R}$} & 844 & 15 & 45 & 3 & 6.55 \\
\hline & & 15 & 54 & 0 & 6.05 \\
\hline \multirow[t]{2}{*}{ Posterior cingulate (BA 29) $-\mathrm{L}$} & 365 & -12 & -48 & 18 & 6.08 \\
\hline & & 6 & -21 & 42 & 6.08 \\
\hline $\operatorname{PrC}(\mathrm{BA} 31)-\mathrm{L}$ & & -6 & -69 & 24 & 5.90 \\
\hline \multirow{2}{*}{ Middle frontal gyrus (BA 6/8)-L } & 194 & -24 & 27 & 45 & 6.59 \\
\hline & & -33 & 9 & 54 & 4.80 \\
\hline Middle temporal gyrus (BA 21) $-\mathrm{L}$ & & -66 & -27 & -12 & 5.84 \\
\hline Fusiform gyrus (BA 20)—L & & -45 & -24 & -15 & 5.79 \\
\hline Caudate-L $\mathrm{L}$ & & -39 & -39 & 3 & 5.63 \\
\hline Insula (BA 13) $-\mathrm{L}$ & 191 & -33 & -18 & 15 & 5.75 \\
\hline Parahippocampal gyrus (BA 30)—L & & -24 & -3 & 6 & 5.47 \\
\hline Caudate-L & & -12 & -9 & 27 & 5.42 \\
\hline Parietal, angular gyrus (BA 39) $-\mathrm{R}$ & 184 & 45 & -63 & 33 & 6.42 \\
\hline Superior occipital gyrus (BA 19) $-R$ & & 42 & -78 & 36 & 5.81 \\
\hline Inferior parietal lobe (BA 40) $-\mathrm{R}$ & & & -60 & 45 & 5.67 \\
\hline Middle frontal gyrus (BA 8) $-\mathrm{R}$ & 160 & 27 & 18 & 42 & 5.96 \\
\hline Medial frontal gyrus (BA 32) $-\mathrm{R}$ & & 18 & 15 & 45 & 5.41 \\
\hline Superior temporal gyrus (BA 22) - R & & 48 & -33 & 6 & 5.75 \\
\hline \multirow[t]{2}{*}{ Middle temporal gyrus (BA 21/22) $-\mathrm{R}$} & & 57 & -30 & 6 & 5.47 \\
\hline & & 60 & -33 & -12 & 5.42 \\
\hline \multirow[t]{2}{*}{ Middle frontal gyrus (BA 10) $-\mathrm{L}$} & 58 & -33 & 57 & 6 & 5.84 \\
\hline & & -42 & 51 & -6 & 5.63 \\
\hline Superior frontal gyrus (BA 10)-L & & -30 & 54 & -6 & 5.79 \\
\hline Parietal lobe (BA 39)—L & 47 & -51 & -60 & 33 & 5.96 \\
\hline Inferior parietal lobe $(\mathrm{BA} 40)-\mathrm{L}$ & & -51 & -57 & 42 & 5.41 \\
\hline Parietal, postcentral gyrus (BA 7) $-R$ & 35 & 18 & -51 & 63 & 5.30 \\
\hline \multirow[t]{2}{*}{ Parietal, postcentral gyrus (BA 5/7) - L } & 33 & -24 & -45 & 72 & 5.61 \\
\hline & & -18 & -54 & 66 & 5.20 \\
\hline $\operatorname{PrC}(\mathrm{BA} 19)-\mathrm{L}$ & 16 & -39 & -72 & 36 & 5.28 \\
\hline $\operatorname{PrC}(\mathrm{BA} 7)-\mathrm{R}$ & 16 & 9 & -69 & 39 & 5.03 \\
\hline Cuneus (BA 19) & & 0 & -81 & 36 & 5.01 \\
\hline \multirow[t]{2}{*}{ Parietal, postcentral gyrus (BA 2) $-\mathrm{R}$} & 14 & 33 & -30 & 36 & 5.00 \\
\hline & & 39 & -30 & 30 & 4.82 \\
\hline Middle temporal gyrus (BA 39) — L & 14 & -33 & -63 & 21 & 5.15 \\
\hline
\end{tabular}


Table 1. (continued)

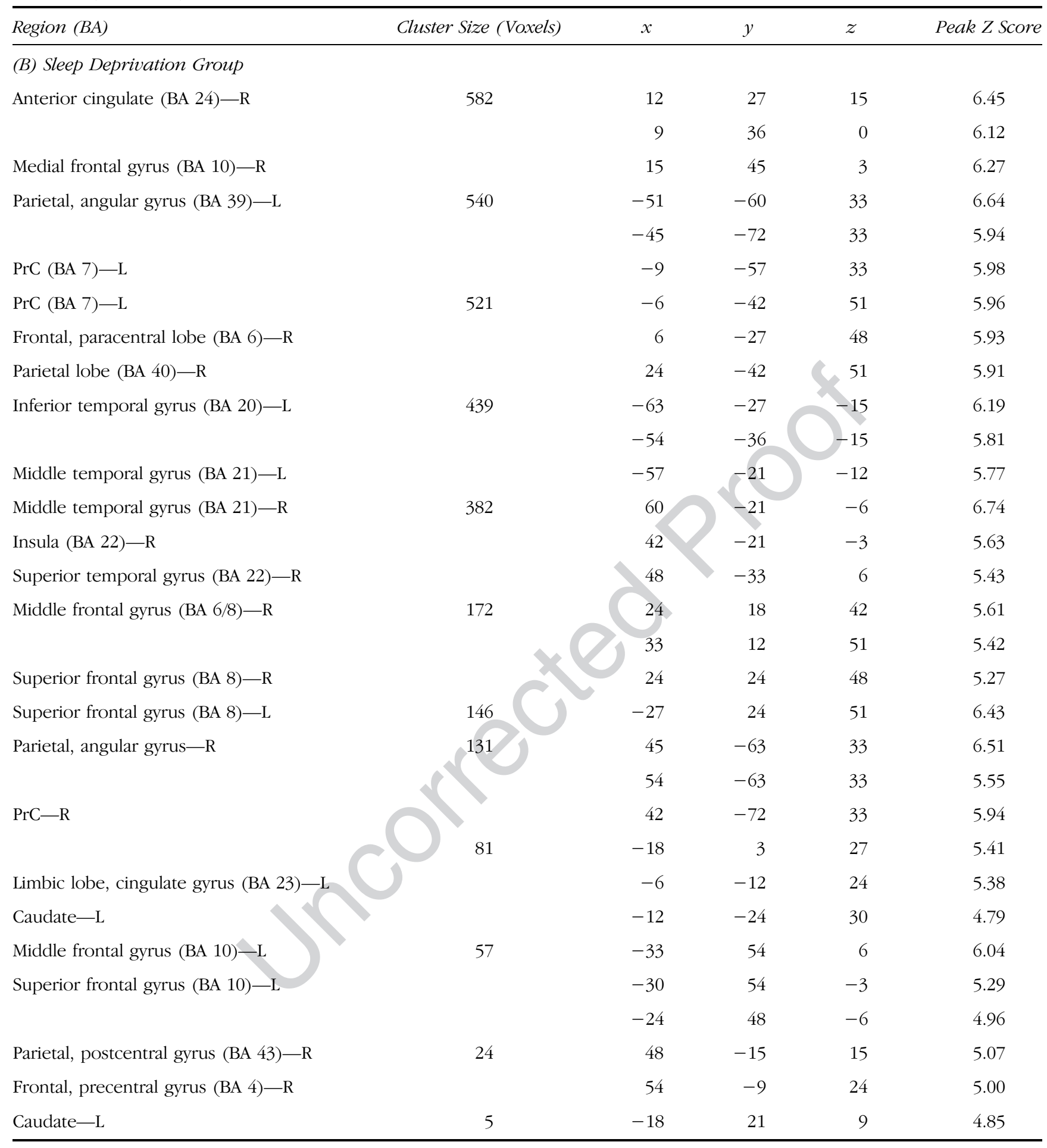

The $x-y-z$ coordinates are given in peak Montreal Neurological Institute space coordinates. L and R denote left and right. The Brodmann's area (BA) location is identified according to the atlas of Talairach and Tournoux (1988).

(Figure 2). Specifically, there was significantly less deactivation in the sleep deprivation group within the dACC region of the default-mode network yet significantly greater deactivation in the PrC for the sleep deprivation group. Therefore, sleep-deprived subjects demonstrated a bidirectional dACC-PrC imbalance in the pattern of task-induced deactivation.
One interpretation of these differences in the deprived group (less task-induced deactivation in anterior areas, greater deactivation in posterior areas) could be a compensatory mechanism. That is, the observed changes reflect adaptive alterations in the context of on-task performance (here, memory encoding). Alternatively, this disparity could reflect dysfunctional brain activity imposed by sleep 
Figure 2. Group-level differences in task-induced deactivation. (A) Significantly less deactivation in the dACC was identified in the sleep-deprived group, relative to the sleep-rested group (upper right panel, cool colors), yet significantly greater deactivation was observed in the $\operatorname{PrC}(\operatorname{PrC})$ in the sleep-deprived group (upper right panel, hot colors; peak Montreal Neurological Institute space coordinates $(x, y, z)$ : $\mathrm{dACC}=6,42,36, Z$ score $=$ 3.73; $\operatorname{PrC}=-6,-42,54$, $Z$ score $=3.74$ ), effects are significant at $p<.001 ;$ $\geq 5$ contiguous voxels.

(B) Corresponding histograms of parameter estimates (effect size) for the dACC and PrC region in the sleep-rested and sleep-deprived groups, representing averaged activity across the peak voxels. Histogram $y$-axis is in arbitrary units (i.e., residual activity after fit of the GLM). Error bars represent $S E ; * p \leq .001$.

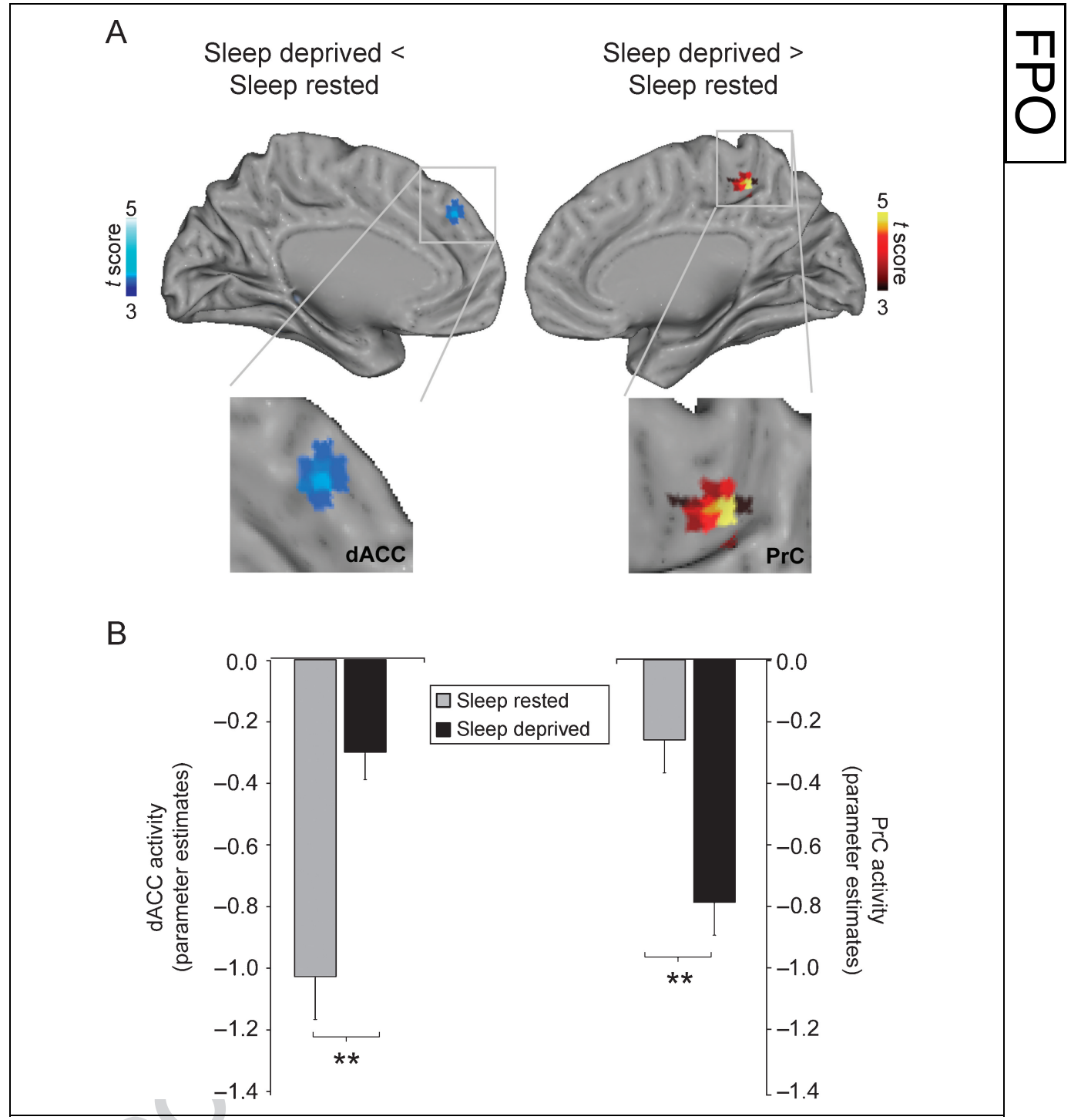

deprivation. To investigate this possibility, we reexamined task-induced deactivations in the ROIs defined in the group level contrast but separated on the basis of successful versus unsuccessful memory encoding trials ("hits" and "misses," respectively). If, for example, the changes observed during sleep deprivation are adaptive, one may expect the greatest relative differences (largest adaptive shift in activity, relative to the control group) to occur during successful memory encoding trials (hits). However, if these changes were maladaptive, one would expect the inverse-the largest relative difference to be expressed between the two groups during failed memory encoding trials (misses).

Although the direction of the differences in task-induced deactivation remained, the magnitude and the significance of these differences were different for successful and unsuccessful encoding trials (Figure 3). Specifically, the difference between the control and the sleep deprivation groups in the anterior and posterior midline regions was most pronounced and significant for misses (failed trials);
$\mathrm{dACC}(p=.0002)$ and $\operatorname{PrC}(p=.0005)$ (Figure 3B). In contrast, during successful trials (hits, Supplementary Table 1A), task-induced deactivation was more proximal between the two groups, showing only a marginal trend toward significant for the dACC $(p=.09)$ and a reduced magnitude of difference (and significance) for the $\operatorname{PrC}$ region $(p=$ .001). Therefore, the imbalance in task-induced deactivation identified in the main group comparison, based on this separation, appears to be more indicative of dysfunction (i.e., was greatest for unsuccessful task trials) rather than attempted compensation. One potential influence contributing to this augmented activity during misses (trials of unsuccessful encoding) could be microsleep episodes. However, this is unlikely for at least two reasons. First, miss trials still received a behavioral response by the subject at the time of encoding - trials that did not receive a response (omit trials) were excluded from the analysis (Supplementary Table 1B). Second, response times, often used as a measure of alertness, were not significantly different between the two groups for miss trials (nor were they for hit trials; 
Supplementary Table 1B). Furthermore, response times for hits and misses within each group were also not significantly different from each other.

Motivated by the growing interest in identifying biomarkers sensitive to indexing sleep loss and prolonged wakefulness (e.g., Gilestro, Tononi, \& Cirelli, 2009; Frey, Fleshner, \& Wright, 2007), we further sought to determine whether differential activation within these two regions alone could accurately dissociate subjects who were sleep deprived from those who were sleep rested. Subjects were plotted in two-dimensional space on the basis of activity in the AACC and PrC. Using principles of receiver operator characteristics (ROCs; Fawcett, 2006) and plotting a diagonal $y=x$ regression line bisecting this two-dimensional space of activity within these anterior and posterior midline regions, we calculated the sensitivity and the specificity for discriminating subjects in each of the two conditions

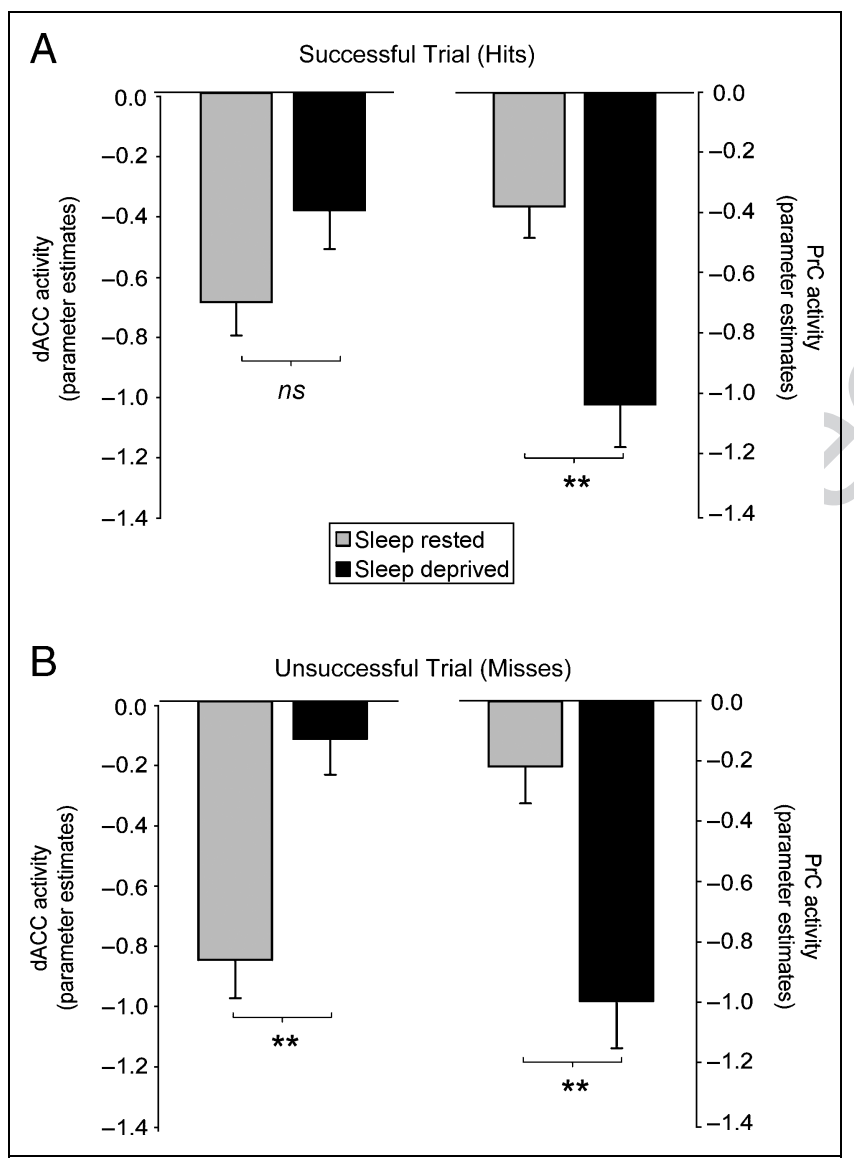

Figure 3. (A) Parameter estimates (effect size) for the dACC and $\operatorname{PrC}$ region in the sleep-rested and sleep-deprived groups for successful trials (hits), representing averaged activity across the peak voxels. Histogram $y$-axis is in arbitrary units (i.e., residual activity after fit of the GLM). Error bars represent $S E$; **p $\leq .001$. (B) Parameter estimates (effect size) for the dACC and PrC region in the sleep-rested and sleep-deprived groups for unsuccessful trials (misses), representing averaged activity across the peak voxels. Histogram $y$-axis is in arbitrary units (i.e., residual activity after fit of the GLM). Error bars represent $S E ; * p \leq .001$

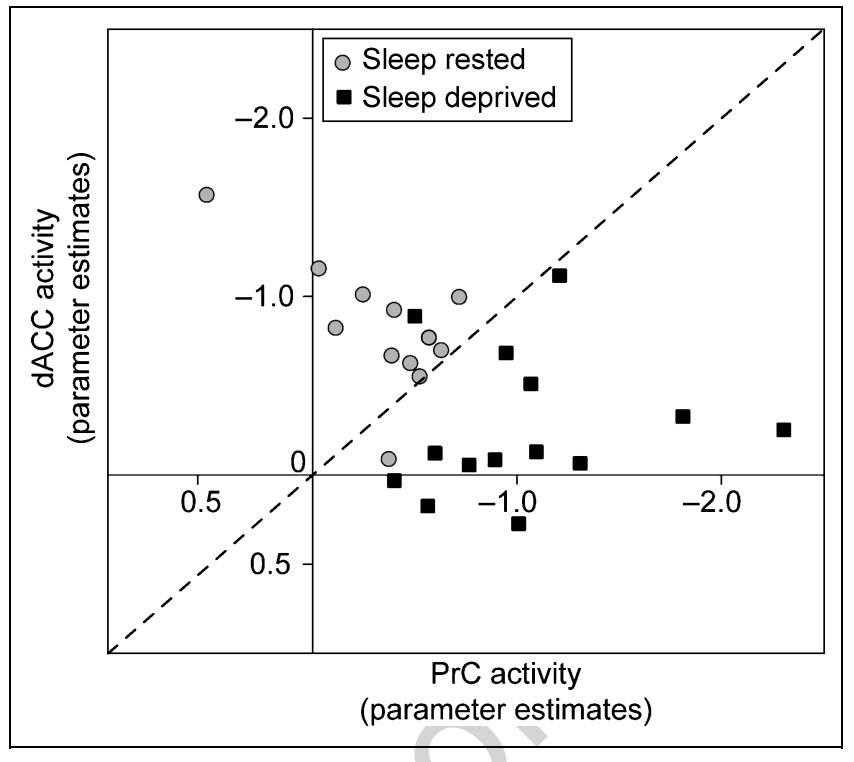

Figure 4. Two-dimensional space plot of individual study participants on the basis of task-induced deactivation (parameter estimates) in the dACC and $\operatorname{PrC}(\operatorname{PrC})$ identified in the group-level comparison. Diagonal dashed line represents $y=x$ segregation used in calculating the ROC power of segregating sleep-deprived from sleep-rested subjects (represented by individual data points).

(Figure 4). Task-related deactivation within the dACC and $\operatorname{PrC}$ was capable of differentiating sleep-deprived individuals from sleep-rested individuals with a $93 \%$ degree of sensitivity (i.e., identifying 13 of 14 sleep-deprived subjects accurately) and a 92\% degree of specificity (by successfully excluding 11 of 12 sleep-rested subjects; Figure 4). Therefore, resting-state activity within these regions alone offered a high degree of discriminatory power in accurately dissociating sleep-deprived from sleep-rested participants.

In addition, within the sleep-rested group, the number of hours of prior reported sleep demonstrated a significant positive correlation with activity in the dACC $(r=0.59$, $p=.04$; the region exhibiting less activity in the deprivation group), and a nonsignificant negative association was evident for the $\operatorname{PrC}(r=-0.47, p=.12$; the region showing significantly more activity in the group with prolonged wakefulness; Figure 5A). In contrast, within the sleep deprivation group, no strong associations were observed between the hours of extended waking and activity within either the dACC or the $\operatorname{PrC}$ regions (both $r<0.29, p>$ .30; Figure 5B). Interestingly, in the sleep-rested group, there was also a relationship in activity between these regions themselves: a negative correlation between the $\mathrm{dACC}$ and the $\operatorname{PrC}(r=-0.67, p=.016$; visualized within Figure $5 \mathrm{~B}$ ) and a reciprocal connection that has previously been reported between the anterior and the posterior default network nodes (Hampson, Driesen, Skudlarski, Gore, \& Constable, 2006). However, no such reciprocity between these regions was observed within the sleep deprivation group $(r=0.12, p=.69)$. 


\section{DISCUSSION}

Here we demonstrate that one night of sleep loss triggers a bidirectional imbalance in midline anterior and posterior brain regions associated with the default-mode network. Furthermore, this imbalance was most significant during failed task trials (unsuccessful memory encoding attempts). In addition, the magnitude of task-induced deactivation within both regions was associated with the amount of obtained prior sleep, and not the extent of continued hours of waking. Therefore, a characteristic signature of the sleep-deprived brain may be the dysregulation not only of on-task brain activity but also of off-task resting-state modes of brain activity.

\section{Task-related Decreases and the Default-mode Network}

Existing neuroimaging studies have consistently highlighted the importance of the dACC and $\mathrm{PrC}$ in the defaultmode network (Fransson \& Marrelec, 2008; Schilbach et al.,

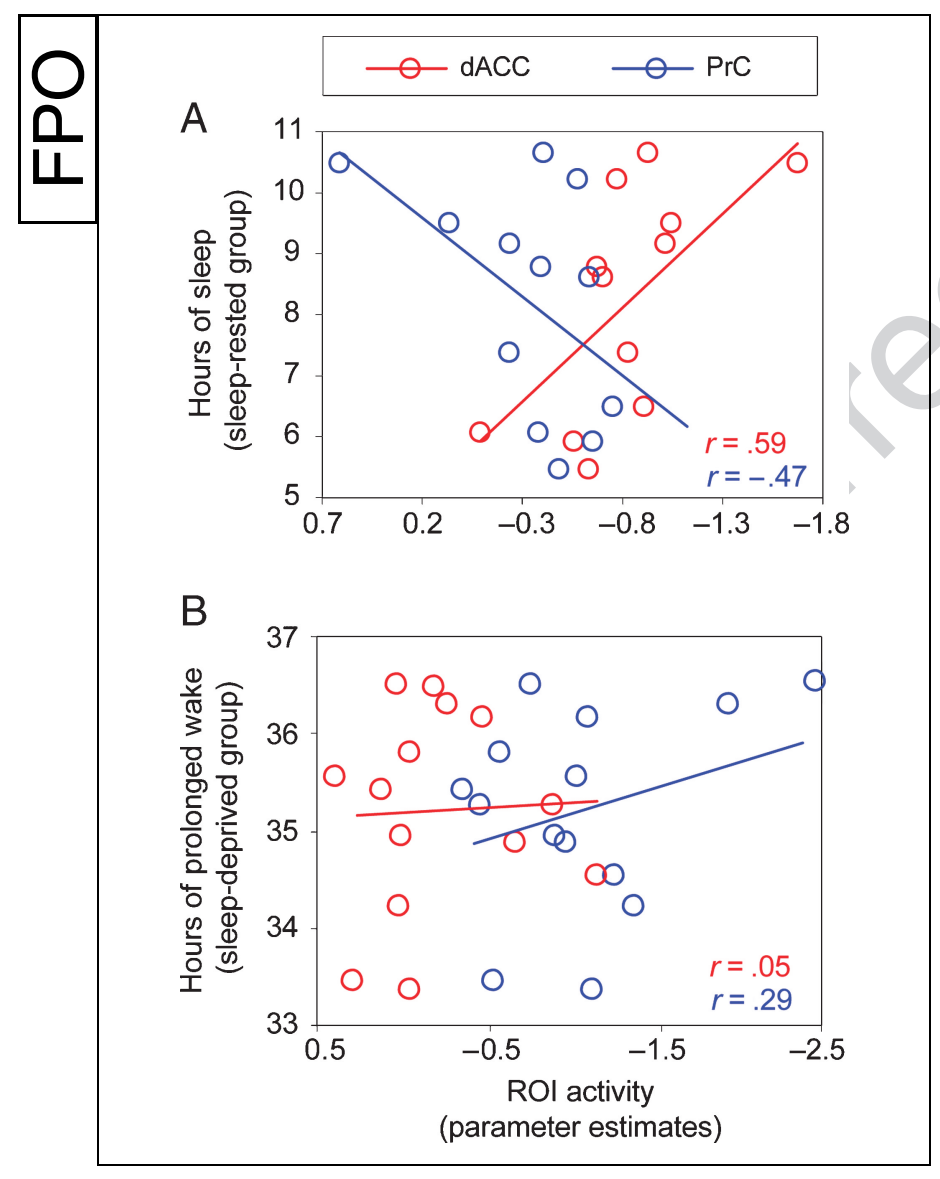

Figure 5. Correlation plots between task-induced deactivation activity (parameter estimates) in the $\mathrm{dACC}$ and $\operatorname{PrC}(\operatorname{PrC})$ identified in the group level contrasts (Figure 2) and (A) hours of prior reported sleep in the sleep-rested group and (B) hours of prolonged waking in the sleep deprivation group. The one subject that could be considered an outlier in the sleep-rested group with strong dACC and $\operatorname{PrC}$ values was within $2 S D$ s of the group mean for both locations.
2008; Seeley et al., 2007; Dosenbach et al., 2006). For example, Dosenbach et al. (2007) have shown that dACC forms part of the "cingulo-opercular" resting-state network related to goal-directed behavior, associated with the proficiency of controlled and stable maintenance of task sets.

Our findings of altered activity within these midline regions may represent a characteristic state of the sleepdeprived resting brain (at least following one night of sleep loss). Moreover, the extent of altered activity within these anterior and posterior default-network nodes was able to accurately distinguished individuals who were deprived of sleep from those who were not with a high degree of accuracy. Although remaining exploratory and necessitating further validation, such findings intimate an additional utility of human neuroimaging techniques in providing sensitive brain biomarkers of the sleep-deprived state (Thomas et al., 2000, 2003; Braun et al., 1997). It will next be informative to examine how such measures track with other brain indices of sleep deprivation, to determine how these alterations cross correlate with measure of cognitive performance, and to evaluate the impact of pharmacological mediators of wake and sleep on these neural markers.

The identified abnormalities of default-mode activity may contribute to the archetypal cognitive hallmark of sleep deprivation-unstable attention (Lim \& Dinges, 2008). Impairment of resting-state activity within this midline network of the default system under conditions of sleep deprivation may prevent the ability to sustain attention, negating maintenance of stable task engagement (Durmer \& Dinges, 2005). It is interesting to note the parallels of such a description and the commonly reported characteristics of the sleepdeprived state: the inability to sustain vigilant, goal-directed task performance (drowsy driving being an ecological example of this danger; Lim \& Dinges, 2008). Using a psychomotor vigilance task, work by Lim and Dinges (2008) and Durmer and Dinges (2005) has elegantly demonstrated that sleep deprivation mitigates the ability to sustain prolonged attentional engagement, resulting in concentration lapses and slowed response times. Moreover, during task trials when performance slows considerably, indicative of decreased attentional ability, Drummond et al. (2005) have demonstrated that activity within midline structures, including anterior cingulate as well as posterior regions, reappears in sleep-deprived subjects and may reflect an uncontrolled reengagement of the resting state.

The PrC/PCC node of the default-mode network, which demonstrated greater task-related deactivation in sleepdeprived group, has been proposed to play a pivotal role in how intrinsic activity is mediated through the defaultmode network (Fransson \& Marrelec, 2008). It is suggested that the PrC forms part of a "fronto-parietal" resting-state network, whose activity is involved in the top-down adjustment and control of task performance, for example, in response to feedback or error signals (Dosenbach et al., $2006,2007)$. When considered in the context of these findings, it would seem reasonable to hypothesize that changes 
in PrC default-mode activity under conditions of sleep deprivation reflect attempted adaptation or compensation responses rather than dysfunction. This would support recent findings of greater task-induced deactivation in sleepdeprived participants during a visual short-term memory task, corresponding to increasing memory load (Chee \& Chuah, 2007). However, our additional subset analysis of successful and unsuccessful trial performance does not support this interpretation, at least for the current task paradigm (episodic memory encoding). Specifically, differences in task-induced deactivations within these midline anterior and posterior default-mode regions were greatest in deprived subjects during failed memory encoding events (miss trials), yet more similar to the control group (and not significantly different for the anterior node) during successful encoding trials. A compensatory explanation of these differences would predict the inverse result, favoring the interpretation of maladaptive dysfunction (Otten \& Rugg, 2001b).

In addition, we also identified a significant correlation between the dACC (and a nonsignificant association with PrC activity) in the sleep control group, yet an absence of such associations in the sleep-deprived group. Interestingly, greater connectivity between the posterior cingulate and the medial prefrontal nodes of the default-mode network has been associated with superior performance during a working memory task (Hampson et al., 2006). Moreover, the breakdown in reciprocal connectivity between the anterior cingulate and the posterior (PrC and PCC) components of the default-mode network has been reported in disease states including attention deficit disorder (Castellanos et al., 2007; Tian et al., 2006). Therefore, it may not only be an imbalance in task-induced deactivation within each of these regions associated with impaired performance in the sleep-deprived state but also a decoupling of the regulation between these nodes.

Whether this pattern of disrupted default-mode activity associated with sleep deprivation is consistent across all cognitive task domains will require further elucidation. It is also important to note that the current paradigm examined task-induced deactivation differences rather than a simple resting-state epoch, a measure that may offer a more unconstrained examination of resting network differences (Horovitz et al., 2008; Long et al., 2008). If confirmed with these alternate assessments of default brain activity, it would imply that canonical and underappreciated factors contributing to neurocognitive impairment under conditions of sleep deprivation are the failure of, and the inability to disengage from, brain networks that persist during rest. Such fMRI measures of resting-state activity also offer a different but complementary visualization of the brain changes caused by sleep deprivation to those obtained using more global PET measure CBF and metabolisms (Thomas et al., 2000, 2003; Braun et al., 1997). Understanding the relationship between global brain metabolism changes caused by sleep deprivation and the more local (regional) alterations obtained using
fMRI represents a fertile future area of investigation (Braun et al., 1997).

\section{Association with Sleep and Extended Wakefulness}

In addition to these between-group differences, the relative balance of deactivation in the dACC node (and to a lesser degree, in the PrC node) correlated with the amount of prior sleep within the control group and not extended time awake in the sleep deprivation group. These associations are supportive of the hypothesis that the metric of sleep loss rather than the measure of continued wakefulness is associated with changes in the dACC and PrC regions that differentiated the sleep-deprived from the sleep-rested subjects. Although the current study does not dissociate whether the differences in brain activity are due to a lack of sleep in the deprivation group or the additional continued hours of waking, it is interesting to speculate, on the basis of the identified correlations, that at least some aspects of the default network are dependent on prior sleep for their stability. This is particularly intriguing on the basis of the direction of these correlations, which demonstrated a positive association between the amount of prior sleep and $\mathrm{dACC}$ activity (the region impaired in the deprivation group), yet a negative, but nonsignificant, correlation with $\operatorname{PrC}$ activity (the region demonstrating increased activation in sleep-deprived subjects). Nevertheless, the measure of sleep time in the current study is self-reported and can only be taken as approximate. It will now be important to measure sleep physiologically and to determine not only whether specific types of sleep (e.g., non-rapid eye movement [NREM] or rapid eye movement) demonstrate associations with resting-state brain activity but also whether the unique characteristics of sleep EEG oscillations (e.g., spectral power, coherence, wave slope, and amplitude) provide additional explanatory power in such analyses.

Among the number of theories addressing the functional role served by the default-mode state, and beyond it intrinsic brain activity as a whole (Buckner et al., 2008; Buckner \& Vincent, 2007; Raichle \& Snyder, 2007), one such hypothesis suggests that resting-state activity supports off-line processing of recently acquired information within the context of preexisting knowledge (Miall \& Robertson, 2006). Such processing may allow for the testing of unique memory associations, and by doing so importantly make flexible predictions about the future (Schacter, Addis, \& Buckner, 2007). In this regard, the role of sleep, and a lack thereof, becomes particularly relevant. For example, a recent report has demonstrated that sleep not only strengthens individual item memories but can actually facilitate the off-line building of distant relational associations between them (Ellenbogen, Hu, Payne, Titone, \& Walker, 2007). Moreover, following initial practice on a problem solving task, a night of sleep significantly increases the ability to gain insight of a hidden rule the following day (Wagner, Gais, Haider, Verleger, \& Born, 2004). However, this next-day insight was not evident immediately upon awaking. Instead, 
it emerged only following substantial additional waking (re)engagement on the task. Furthermore, participants who were sleep deprived and retested in the deprivation state failed to gain such creative insight.

Findings such as these suggest that sleep may not only facilitate ongoing patterns of resting-state activity at night (Fukunaga et al., 2006) but additionally support its integrity for internal processing of information the next day, potentially identifying relational associations among previously acquired knowledge. Moreover, without adequate prior sleep, this mode of brain operation may be unable to operate efficiently. Future investigations of this kind may lead to the appreciation of a related interdependence between sleep states at night and waking default activity the next day, linking a common function that has been assigned to both-bomeostasis (Boly et al., 2008; Tononi \& Cirelli, 2006). Several theories suggest that sleep represents an ideal neurophysiological state for achieving the homeostatic balance of synaptic connectivity within the brain (Tononi \& Cirelli, 2003, 2006; Sejnowski \& Destexhe, 2000; Crick \& Mitchison, 1983). A number of recent studies have specifically highlighted commonalities between NREM slow-wave sleep (SWS) and aspects of resting-state networks, including NREM SWS functional anatomy (DangVu et al., 2008), the slow oscillation brain characteristics of NREM SWS fMRI and EEG signals, and the neural sites that appear to generate NREM SWS (Murphy et al., 2009; Boly et al., 2008). It is also interesting to note that a similar hypothesis has been suggested for intrinsic resting-state brain activity in sculpting new circuits and/or maintaining the balance of those already in existence (Raichle \& Snyder, 2007). It would therefore appear that canonical states of intrinsic neural activity represent an organizing principal of brain function, which may be observed during both wake and sleep, each of which may be symbiotically related to the other and may even share some common functional $\operatorname{goal}(\mathrm{s})$.

\section{Acknowledgments}

The authors thank Dr. Edwin Robertson for his insightful and helpful comments regarding these findings and Heather O'Leary for technical MRI assistance. This work was supported in part by grants from the National Institutes of Health (MH69,935 [M. P. W.] and NS48,242 [S.-S. Y.]) and the American Academy of Sleep Medicine (M. P. W.).

Reprint requests should be sent to Matthew P. Walker, Department of Psychology, University of California, Tolman Hall 3331, Berkeley, CA 94720-1650, or via e-mail: mpwalker@berkeley.edu.

\section{REFERENCES}

Balduzzi, D., Riedner, B. A., \& Tononi, G. (2008). A BOLD window into brain waves. Proceedings of the National Academy of Sciences, U.S.A., 105, 15641-15642.

Binder, J. R., Frost, J. A., Hammeke, T. A., Bellgowan, P. S., Rao, S. M., \& Cox, R. W. (1999). Conceptual processing during the conscious resting state. A functional MRI study. Journal of Cognitive Neuroscience, 11, 80-95.
Boly, M., Phillips, C., Tshibanda, L., Vanhaudenhuyse, A., Schabus, M., Dang-Vu, T. T., et al. (2008). Intrinsic brain activity in altered states of consciousness: How conscious is the default mode of brain function? Annals of the New York Academy of Sciences, 1129, 119-129.

Braun, A. R., Balkin, T. J., Wesensten, N. J., Carson, R. E., Varga, M., Baldwin, P., et al. (1997). Regional cerebral blood flow throughout the sleep-wake cycle. An H215O PET study. Brain, 120, 1173-1197.

Buchel, C., Holmes, A. P., Rees, G., \& Friston, K. J. (1998). Characterizing stimulus-response functions using nonlinear regressors in parametric fMRI experiments. Neuroimage, 8, 140-148.

Buckner, R. L., Andrews-Hanna, J. R., \& Schacter, D. L. (2008). The brain's default network: Anatomy, function, and relevance to disease. Annals of the New York Academy of Sciences, 1124, 1-38.

Buckner, R. L., \& Vincent, J. L. (2007). Unrest at rest: Default activity and spontaneous network correlations. Neuroimage, 37, 1091-1096; discussion 1097-1099.

Castellanos, F. X., Margulies, D. S., Kelly, C., Uddin, L. Q., Ghaffari, M., Kirsch, A., et al. (2007). Cingulate-precuneus interactions: A new locus of dysfunction in adult attention-deficit/ hyperactivity disorder. Biological Psychiatry.

Chee, M. W., \& Chuah, Y. M. (2007). Functional neuroimaging and behavioral correlates of capacity decline in visual short-term memory after sleep deprivation. Proceedings of the National Academy of Sciences, U.S.A., 104, 9487-9492.

Chee, M. W. L., \& Chuah, L. Y. M. (2008). Functional neuroimaging insights into how sleep and sleep deprivation affect memory and cognition. Current Opinion in Neurology, 21, 417-423.

Cherkassky, V. L., Kana, R. K., Keller, T. A., \& Just, M. A. (2006). Functional connectivity in a baseline resting-state network in autism. NeuroReport, 17, 1687-1690.

Crick, F., \& Mitchison, G. (1983). The function of dream sleep. Nature, 304, 111-114.

Damoiseaux, J. S., Beckmann, C. F., Arigita, E. J., Barkhof, F., Scheltens, P., Stam, C. J., et al. (2007). Reduced resting-state brain activity in the "default network" in normal aging. Cerebral Cortex.

Dang-Vu, T. T., Schabus, M., Desseilles, M., Albouy, G., Boly, M., Darsaud, A., et al. (2008). Spontaneous neural activity during human slow wave sleep. Proceedings of the National Academy of Sciences, U.S.A., 105, 15160-15165.

Davis, S. W., Dennis, N. A., Daselaar, S. M., Fleck, M. S., \& Cabeza, R. (2008). Que PASA? The posterior-anterior shift in aging. Cerebral Cortex, 18, 1201-1209.

Dosenbach, N. U., Fair, D. A., Miezin, F. M., Cohen, A. L., Wenger, K. K., Dosenbach, R. A., et al. (2007). Distinct brain networks for adaptive and stable task control in humans. Proceedings of the National Academy of Sciences, U.S.A., 104, 11073-11078.

Dosenbach, N. U., Visscher, K. M., Palmer, E. D., Miezin, F. M., Wenger, K. K., Kang, H. C., et al. (2006). A core system for the implementation of task sets. Neuron, 50, 799-812.

Drummond, S. P., Bischoff-Grethe, A., Dinges, D. F., Ayalon, L., Mednick, S. C., \& Meloy, M. J. (2005). The neural basis of the psychomotor vigilance task. Sleep, 28, 1059-1068.

Drummond, S. P., \& Brown, G. G. (2001). The effects of total sleep deprivation on cerebral responses to cognitive performance. Neuropsychopharmacology, 25(Suppl. 5), S68-S73.

Drummond, S. P., Brown, G. G., Gillin, J. C., Stricker, J. L., Wong, E. C., \& Buxton, R. B. (2000). Altered brain response to verbal learning following sleep deprivation. Nature, 403, 655-657.

Durmer, J. S., \& Dinges, D. F. (2005). Neurocognitive consequences of sleep deprivation. Seminars in Neurology, 25, 117-129. 
Ellenbogen, J., Hu, P., Payne, J. D., Titone, D., \& Walker, M. P. (2007). Human relational memory requires time and sleep. Proceedings of the National Academy of Sciences, U.S.A., 104, 7723-7728.

Fair, D. A., Schlaggar, B. L., Cohen, A. L., Miezin, F. M., Dosenbach, N. U., Wenger, K. K., et al. (2007). A method for using blocked and event-related fMRI data to study "resting state" functional connectivity. Neuroimage, 35, 396-405.

Fawcett, T. (2006). An introduction to ROC analysis. Pattern Recognition Letters, 27, 861-874.

Fox, M. D., \& Raichle, M. E. (2007). Spontaneous fluctuations in brain activity observed with functional magnetic resonance imaging. Nature Reviews Neuroscience, 8, 700-711.

Fransson, P., \& Marrelec, G. (2008). The precuneus/posterior cingulate cortex plays a pivotal role in the default mode network: Evidence from a partial correlation network analysis. Neuroimage, 42, 1178-1184.

Frey, D. J., Fleshner, M., \& Wright, K. P., Jr. (2007). The effects of 40 hours of total sleep deprivation on inflammatory markers in healthy young adults. Brain, Behavior, and Immunity, 21, 1050-1057.

Friston, K., Holmes, A., Worsley, K., Poline, J., Frith, C., \& Frackowiak, R. (1995). Statistical parametric maps in functional imaging: A general linear approach. Human Brain Mapping, 2, 189-210.

Friston, K. J., Fletcher, P., Josephs, O., Holmes, A., Rugg, M. D., \& Turner, R. (1998). Event-related fMRI: Characterizing differential responses. Neuroimage, 7, 30-40.

Fukunaga, M., Horovitz, S. G., van Gelderen, P., de Zwart, J. A., Jansma, J. M., Ikonomidou, V. N., et al. (2006). Large-amplitude, spatially correlated fluctuations in BOLD fMRI signals during extended rest and early sleep stages. Magnetic Resonance Imaging, 24, 979-992.

Garrity, A. G., Pearlson, G. D., McKiernan, K., Lloyd, D., Kiehl, K. A., \& Calhoun, V. D. (2007). Aberrant "default mode" functional connectivity in schizophrenia. American Journal of Psychiatry, 164, 450-457.

Gilestro, G. F., Tononi, G., \& Cirelli, C. (2009). Widespread changes in synaptic markers as a function of sleep and wakefulness in Drosophila. Science, 324, 109-112.

Greicius, M. D., Srivastava, G., Reiss, A. L., \& Menon, V. (2004). Default-mode network activity distinguishes Alzheimer's disease from healthy aging: Evidence from functional MRI. Proceedings of the National Academy of Sciences, U.S.A., 101, 4637-4642.

Gusnard, D. A., Akbudak, E., Shulman, G. L., \& Raichle, M. E. (2001). Medial prefrontal cortex and self-referential mental activity: Relation to a default mode of brain function. Proceedings of the National Academy of Sciences, U.S.A., 98, 4259-4264.

Hampson, M., Driesen, N. R., Skudlarski, P., Gore, J. C., \& Constable, R. T. (2006). Brain connectivity related to working memory performance. Journal of Neuroscience, 26, 13338-13343.

Horovitz, S. G., Fukunaga, M., de Zwart, J. A., van Gelderen, P., Fulton, S. C., Balkin, T. J., et al. (2008). Low frequency BOLD fluctuations during resting wakefulness and light sleep: A simultaneous EEG-fMRI study. Human Brain Mapping, 29, 671-682.

Lim, J. L., \& Dinges, D. F. (2008). Sleep deprivation and vigilant attention. Annals of the New York Academy of Sciences, 1129, 305-322.

Linden, A. (2006). Measuring diagnostic and predictive accuracy in disease management: An introduction to receiver operating characteristic (ROC) analysis. Journal of Evaluation in Clinical Practice, 12, 132-139.

Long, X. Y., Zuo, X. N., Kiviniemi, V., Yang, Y., Zou, Q. H., Zhu, C. Z., et al. (2008). Default mode network as revealed with multiple methods for resting-state functional MRI analysis. Journal of Neuroscience Methods, 171, 349-355.

Mazoyer, B., Zago, L., Mellet, E., Bricogne, S., Etard, O., Houde, O., et al. (2001). Cortical networks for working memory and executive functions sustain the conscious resting state in man. Brain Research Bulletin, 54, 287-298.

McKiernan, K. A., Kaufman, J. N., Kucera-Thompson, J., \& Binder, J. R. (2003a). A parametric manipulation of factors affecting task-induced deactivation in functional neuroimaging. Journal of Cognitive Neuroscience, 15, 394-408.

McKiernan, K. A., Kaufman, J. N., Kucera-Thompson, J., \& Binder, J. R. (2003b). A parametric manipulation of factors affecting task-induced deactivation in functional neuroimaging. Journal of Cognitive Neuroscience, 15, 394-408.

Miall, R. C., \& Robertson, E. M. (2006). Functional imaging: Is the resting brain resting? Current Biology, 16, R998-R1000.

Murphy, M., Riedner, B. A., Huber, R., Massimini, M., Ferrarelli, F., \& Tononi, G. (2009). Source modeling sleep slow waves. Proceedings of the National Academy of Sciences, U.S.A., 106, 1608-1613.

Otten, L. J., \& Rugg, M. D. (2001a). Task-dependency of the neural correlates of episodic encoding as measured by fMRI. Cerebral Cortex, 11, 1150-1160.

Otten, L. J., \& Rugg, M. D. (2001b). When more means less Neural activity related to unsuccessful memory encoding. Current Biology, 11, 1528-1530.

Raichle, M. E., MacLeod, A. M., Snyder, A. Z., Powers, W. J., Gusnard, D. A., \& Shulman, G. L. (2001). A default mode of brain function. Proceedings of the National Academy of Sciences, U.S.A., 98, 676-682.

Raichle, M. E., \& Snyder, A. Z. (2007). A default mode of brain function: A brief history of an evolving idea. Neuroimage, 37, 1083-1090; discussion 1097-1089.

Schacter, D. L., Addis, D. R., \& Buckner, R. L. (2007). Remembering the past to imagine the future: The prospective brain. Nature Reviews Neuroscience, 8, 657-661.

Schilbach, L., Eickhoff, S. B., Rska-Jagiela, A. R., Fink, G. R., \& Vogeley, K. (2008). Minds at rest? Social cognition as the default mode of cognizing and its putative relationship to the "default system" of the brain. Consciousness and Cognition, 17, 457-467.

Seeley, W. W., Menon, V., Schatzberg, A. F., Keller, J., Glover, G. H., Kenna, H., et al. (2007). Dissociable intrinsic connectivity networks for salience processing and executive control. Journal of Neuroscience, 27, 2349-2356.

Sejnowski, T. J., \& Destexhe, A. (2000). Why do we sleep? Brain Research, 886, 208-223.

Shulman, G. L., Corbetta, M., Buckner, R. L., Raichle, M. E., Fiez, J. A., Miezin, F. M., et al. (1997). Top-down modulation of early sensory cortex. Cerebral Cortex, 7, 193-206.

Shulman, G. L., Fiez, J. A., Corbetta, M., Buckner, R. L. Miezin, F. M., Raichle, M. E., et al. (1997). Common blood flow changes across visual tasks .2. Decreases in cerebral cortex. Journal of Cognitive Neuroscience, 9, 648-663.

Sorg, C., Riedl, V., Muhlau, M., Calhoun, V. D., Eichele, T., Laer, L., et al. (2007). Selective changes of resting-state networks in individuals at risk for Alzheimer's disease. Proceedings of the National Academy of Sciences, U.S.A., 104, 18760-18765.

Talairach, J., \& Tournoux, P. (1988). Co-planar sterotaxic atlas of the buman brain. New York: Thieme.

Thomas, M., Sing, H., Belenky, G., Holcomb, H., Mayberg, H., Dannals, R., et al. (2000). Neural basis of alertness and cognitive performance impairments during sleepiness: I. Effects of $24 \mathrm{~h}$ of sleep deprivation on waking human regional brain activity. Journal of Sleep Research, 9, 335-352. 
Thomas, M., Sing, H., Belenky, G., Holcomb, H., Mayberg, H., Dannals, R., et al. (2003). Neural basis of alertness and cognitive performance impairments during sleepiness: II. Effects of 48 and $72 \mathrm{~h}$ of sleep deprivation on waking human regional brain activity. Thalamus \& Related Systems, 2, 199-229.

Tian, L., Jiang, T., Wang, Y., Zang, Y., He, Y., Liang, M., et al. (2006). Altered resting-state functional connectivity patterns of anterior cingulate cortex in adolescents with attention deficit hyperactivity disorder. Neuroscience Letters, 400, 39-43.

Tononi, G., \& Cirelli, C. (2003). Sleep and synaptic homeostasis: A hypothesis. Brain Research Bulletin, 62, 143-150.
Tononi, G., \& Cirelli, C. (2006). Sleep function and synaptic homeostasis. Sleep Medicine Reviews, 10, 49-62.

Wagner, U., Gais, S., Haider, H., Verleger, R., \& Born, J. (2004). Sleep inspires insight. Nature, 427, 352-355.

Walker, M. P., \& Stickgold, R. (2006). Sleep, memory and plasticity. Annual Review of Psychology, 10, 139-166.

Yoo, S. S., Hu, P. T., Gujar, N., Jolesz, F. A., \& Walker, M. P. (2007). A deficit in the ability to form new human memories without sleep. Nature Neuroscience, 10, 385-392.

Zhao, X. H., Wang, P. J., Li, C. B., Hu, Z. H., Xi, Q., Wu, W. Y., et al. (2007). Altered default mode network activity in patient with anxiety disorders: An fMRI study. European Journal Radiology, 63, 373-378. 